\title{
A Procedure for Inducing the Occurrence of Rice Seedling Blast in Paddy Field
}

\author{
Peng Qin ${ }^{1 \dagger}$, Xiaochun $\mathrm{Hu}^{2 \dagger}$, Nan Jiang ${ }^{1,2 \dagger}$, Zhenan $\mathrm{Bai}^{2}$, Tiangang Liu ${ }^{2}$, Chenjian $\mathrm{Fu}^{2}$, Yongbang Song ${ }^{2}$, \\ Kai Wang ${ }^{2 *}$, and Yuanzhu Yang ${ }^{1,2 *}$ \\ ${ }^{1}$ College of Agronomy, Hunan Agricultural University, Changsha 410128, China \\ ${ }^{2}$ Key Laboratory of Southern Rice Innovation \& Improvement, Ministry of Agriculture and Rural Affairs, Hunan \\ Engineering Laboratory of Disease and Pest Resistant Rice Breeding, Yuan Longping High-Tech Agriculture Co., Ltd., \\ Changsha 410128, China
}

(Received on December 1, 2020; Revised on February 26, 2021; Accepted on March 1, 2021)

Rice blast caused by the filamentous fungus Magnaporthe oryzae, is arguably the most devastating rice disease worldwide. Development of a high-throughput and reliable field blast resistance evaluation system is essential for resistant germplasm screening, resistance genes identification and resistant varieties breeding. However, the occurrence of rice blast in paddy field is easily affected by various factors, particularly lack of sufficient inoculum, which always leads to the nonuniform occurrence and reduced disease severity. Here, we described a procedure for adequately inducing the occurrence of rice seedling blast in paddy field, which involves pretreatment of diseased straw, initiation of seedling blast for the first batch of spreader population, inducing the occurrence of the second batch of spreader population and test materials. This procedure enables uniform and consistent infection, which facilitates efficient and accurate assessment of seedling blast resistance for diverse rice materials.

Keywords : inoculum, nursery, Oryza sativa, resistance, seedling blast

\footnotetext{
${ }^{\dagger}$ These authors contributed equally to this work.

*Co-corresponding authors.

K. Wang

Phone, FAX) +86-731-87059159

E-mail)wk8587@163.com

Y. Yang

Phone, FAX) +86-731-87059159

E-mail)yzhuyah@163.com

(c) This is an Open Access article distributed under the terms of the Creative Commons Attribution Non-Commercial License (http:// creativecommons.org/licenses/by-nc/4.0) which permits unrestricted noncommercial use, distribution, and reproduction in any medium, provided the original work is properly cited.
}

Articles can be freely viewed online at www.ppjonline.org.
Handling Editor : Jungkwan Lee

Rice (Oryza sativa L.) is one of the most important crops and over half of the world's population consume it as the staple energy source (Fones et al., 2020). Rice blast caused by the fungal pathogen Magnaporthe oryzae (anamorph: Pyricularia oryzae) frequently destroys rice production worldwide, posing a significant risk to global food security (Skamnioti and Gurr, 2009). Annual yield loss due to blast is sufficient to feed 60 million people (Talbot, 2003). Among different strategies for rice blast management, incorporation of resistant $(R)$ genes into rice cultivars through phenotyping complemented with marker-assisted selection (MAS) is generally considered to be one of the most effective, economical and environmentally sustainable (Liang et al., 2017). The occurrence and spread of rice blast disease are significantly influenced by various environmental factors including temperature and humidity (Calvero et al., 1996; Katsantonis et al., 2017). The optimum conditions for infection and spread of the rice blast fungus are at the temperature ranging from $25^{\circ} \mathrm{C}$ to $28^{\circ} \mathrm{C}$ and at the relative humidity over $90 \%$ (Peng et al., 2019). Therefore, artificial inoculation in controlled environments has been widely used for identification of rice blast $R$ genes and dissecting the interaction between rice and M. oryzae in the last two decades. However, it is not applicable in large-scale blast resistance evaluation of germplasm and breeding materials due to its high cost and low-throughput. Natural disease nurseries, usually found in hotspots of rice blast with relatively high disease pressure, enable large-scale resistance evaluation and facilitate breeding for rice blast resistance (Vasudevan et al., 2014). Dawei Mountain Rice Breeding Station (DMRBS) was established by Yuan Longping High-Tech Agriculture Co., Ltd. in Dawei Mountain, Liuy- 
ang city, Hunan province, China in 2001. Development of hybrid rice combinations with broad-spectrum resistance to blast disease is the main aim of DMRBS. Additionally, as one of the three officially authorized rice blast nurseries by Department of Agriculture and Rural Affairs of Hunan Province, DMRBS undertakes the blast resistance evaluation of rice cultivars applied for commercial release in Hunan. It is located at an elevation of $400 \mathrm{~m}$ above sea level. The annual average temperature is $17.3^{\circ} \mathrm{C}$ and it is $28.9^{\circ} \mathrm{C}$ in July, the hottest month during the rice growing season. The annual average rainfall is $1,800-2,000 \mathrm{~mm}$ and relative humidity is over $83 \%$. It has about 1,600 sunshine hours annually (Peng et al., 2019). These environmental conditions are favorable for rice blast epidemics. However, the large-scale and uniform occurrence of rice blast relying solely on natural infection is always unstable due to insufficient inoculum. In previous studies, diseased straw as the primary inducer was sprinkled over the highly susceptible cultivars, then the infected plants could provide abundant inoculum (Jiang et al., 2012, 2020; Sun et al., 2013). However, the diseased straw on spreader plants is easily affected by wind and rain, and may fall into the irrigation water and drift away, which cannot provide sufficient primary inoculum. Additionally, spreader population served as the major source of inoculum may not be fully infected due to the unfavorable environmental conditions in the field and stored conditions of the diseased straw (Peng et al., 2019; Ravelo- son et al., 2017), which usually leads to the non-uniform infection and reduced disease severity of the test materials. To solve this problem, we developed a procedure with two rounds of disease induction, which could provide abundant inoculum and adequately induce the occurrence of rice seedling blast in paddy field. The diseased straw collected in previous rice growing season was dried and stored in a ventilated room. Before use, the diseased straw was first immersed in water for $2 \mathrm{~h}$ and stacked at $28-30^{\circ} \mathrm{C}$ for $24 \mathrm{~h}$, with over $80 \%$ relative humidity. After that, the straw was evenly distributed on a plastic film for $24 \mathrm{~h}$ under the same conditions (Fig. 1A). Several highly susceptible cultivars of different types constitute a spreader population. In our study, highly susceptible cultivars Xiangaizao 7 (an earlyseason variety), LP2300 (a breeding line), and Xiangwanxian 11 (a late-season variety) were selected and uniformly mixed as spreader population. The three cultivars were also used as susceptible checks in the evaluation. The first batch of spreader population was sown 3 weeks before the second batch and the test materials (Fig. 1B). Seven days later, every 10 pieces of pretreated diseased-straw tied to a stick was inserted into the soil of nursery beds at a spacing of about $1 \mathrm{~m}$ instead of sprinkling them on the spreader population (Fig. 1C). The second batch of spreader population as border lines were densely sown together with test materials on nursery beds following a ratio of 6:1 (Fig. 1D). Seven days after sowing, every 6 infected plants from

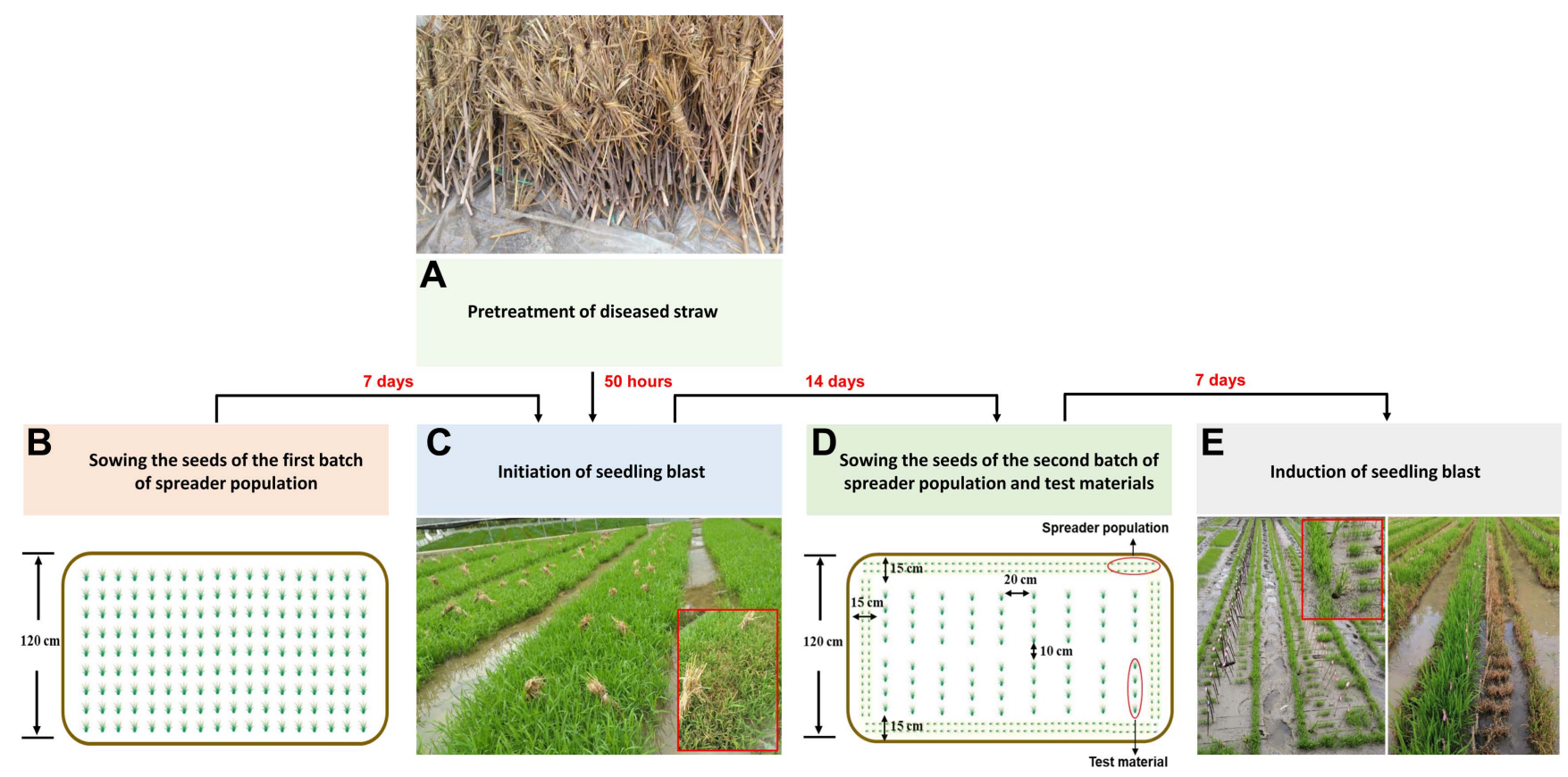

Fig. 1. The flowchart for the induction of rice seedling blast. Pretreatment of diseased straw (A). Sowing the seeds (B) and initiating seedling blast of the first batch of spreader population (C). Sowing the seeds (D) and inducing seedling blast of the second batch of spreader population and test materials (E). 
the first batch of spreader population was transferred to the place close to the border lines at a spacing of $50 \mathrm{~cm}$, which could provide more fresh $M$. oryzae spores to infect the border lines compared with sprinkled diseased-straw (Fig. 1E). Nitrogen was applied as urea at $150 \mathrm{~kg} / \mathrm{ha}$ at 2- to 3 -seedling stage. It is forbidden to use any fungicide. Other field management followed normal agricultural practices.

Based on the developed procedure, a total of 730 indica hybrid rice combinations, which were developed by different companies and breeding institutes and applied for commercial release in Hunan province, were evaluated for seedling blast resistance in DMRBS during 2015-2019. Disease symptoms were scored with a 0-9 rating scale following the Methods of Identification and Evaluation of Rice Resistance to Magnaporthe grisea (Hunan Insititute of Plant Protection, 2006) and further graded as highly resistant (score 0), resistant (scores 1 and 2), moderately resistant (score 3), moderately susceptible (scores 4 and 5), susceptible (scores 6 and 7), and highly susceptible (scores 8 and 9). The results indicated that around $84 \%$ of the hybrid combinations showed moderate susceptibility to high susceptibility (Fig. 2). Only $0.96 \%$ and $15.07 \%$ of the hybrid combinations conferred resistance and moderate resistance, respectively. No combination was found to be highly resistant. However, the percentages of resistant and moderately resistant hybrid combinations were higher during 2017-2019 due to more attention paid to the genetic improvement of rice blast resistance by breeders in recent years (Fig. 2).

Large-scale phenotypic screening based on the procedure in combination with MAS of resistance genes, enabled the

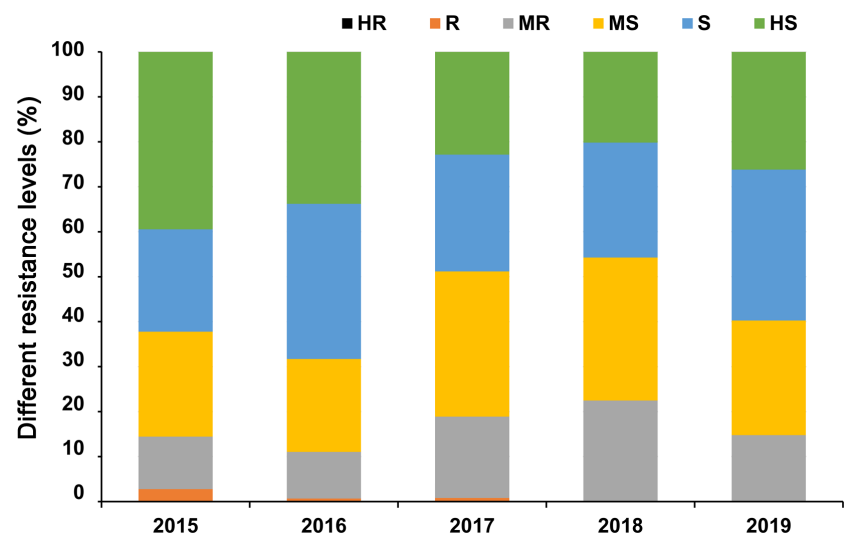

Fig. 2. The distribution of seedling blast resistance of the 730 indica hybrid rice combinations applied for commercial release during 2015-2019. HR, highly resistant; R, resistant; MR, moderately resistant; MS, moderately susceptible, S, susceptible; HS, highly susceptible.

percentage of resistant breeding materials in our company to increase gradually from $4.76 \%$ to more than $70 \%$ over the period 2008-2019 (Fig. 3). By using the procedure in DMRBS, a total of 280 indica hybrid rice combinations with blast resistance (integrated disease index $\leq 4.0$ ) have been certified for commercial release in different southern regions of China since 2015, accounting for $71.6 \%$ of our released hybrid combinations.

In summary, we report a procedure for adequately inducing the occurrence of seedling blast. Instead of sprinkling, the diseased straw tied to a stick was inserted into the soil of nursery beds, which could consistently and stably provide primary inoculum. Two rounds of disease induction

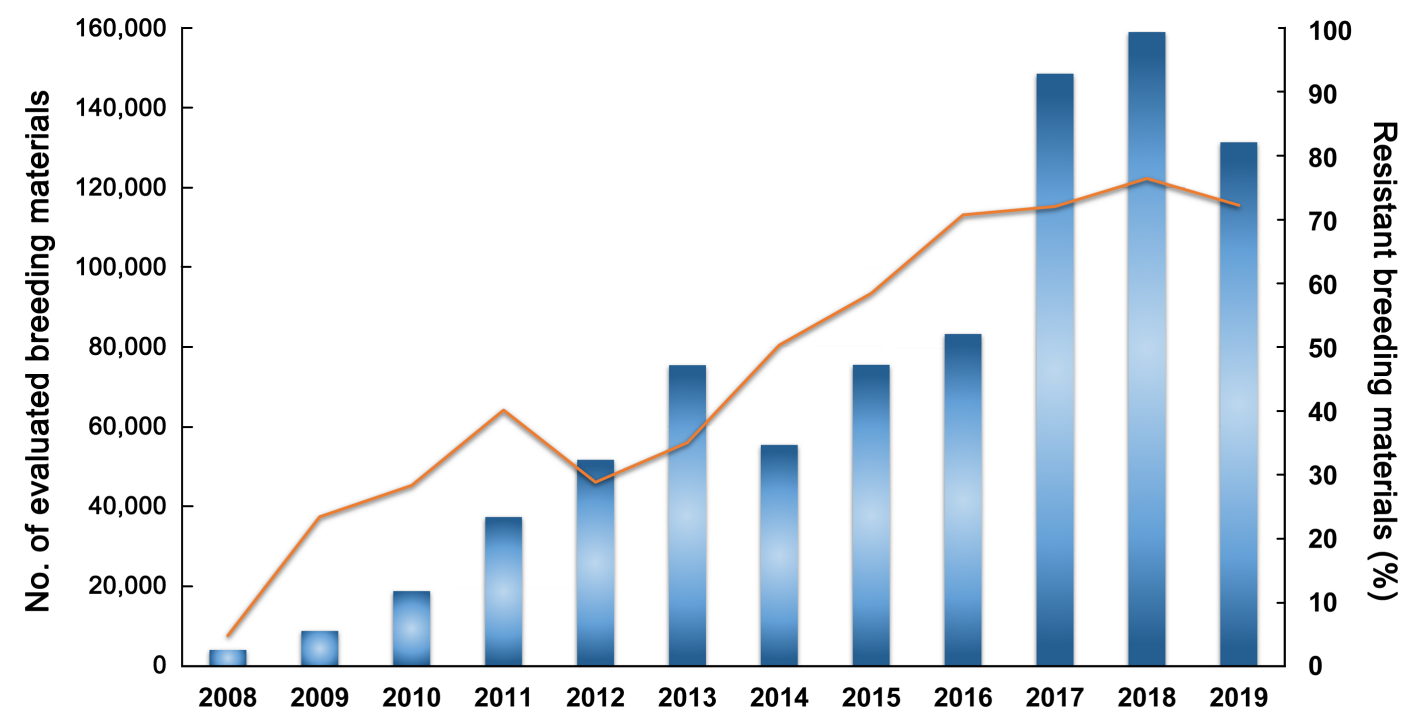

Fig. 3. Number of breeding materials evaluated in Dawei Mountain Rice Breeding Station (DMRBS) during 2008-2019 (bars, left yaxis) and the percentage of resistant breeding materials (line, right y-axis). 
for the spreader population are very important for providing sufficient inoculum to infect the test materials. Following the procedure, we conducted a large-scale seedling blast resistance evaluation for the indica hybrid rice combinations applied for commercial release in Hunan province. The results revealed that most of the hybrid combinations were susceptible. Through efficient phenotypic screening in DMRBS combining with MAS, multiple valuable resistance genes including Pi2, Pigm, Pil, and Pita were introduced into our core breeding materials and a series of hybrid rice combinations with blast resistance have been developed and released. For example, a total of 93 LongLiangyou and Jing-Liangyou hybrid rice combinations derived from the mid-season indica thermo-sensitive genic male sterile lines Longke638S and Jing4155S, respectively, were nationally certified during 2015-2019 and these hybrid combinations carry $R$ genes ranging from 3 to 7. About $71.2 \%, 82.3 \%, 10.7 \%$, and $5.7 \%$ of the combinations contain Pi2, Pita, Pil, and Pigm, respectively. The procedure facilitates our breeding for blast resistance.

\section{Conflicts of Interest}

No potential conflict of interest relevant to this article was reported.

\section{Acknowledgments}

This work was supported by the Special Project for Science and Technology Talents of Hunan (2019RS2054), State Key Laboratory of Hybrid Rice (Hunan Hybrid Rice Research Center) Open Project Fund (2018KF03), Science and Technology Innovation Program of Hunan (2018NK1020).

\section{References}

Calvero, J. S. Jr., Coakley, S. M. and Teng, P. S. 1996. Development of empirical forecasting models for rice blast based on weather factors. Plant Pathol. 45:667-678.
Fones, H. N., Bebber, D. P., Chaloner, T. M., Kay, W. T., Steinberg, G. and Gurr, S. J. 2020. Threats to global food security from emerging fungal and oomycete crop pathogens. Nat. Food 1:332-342.

Hunan Insititute of Plant Protection. 2006. Methods of identification and evaluation of rice resistance to Magnaporthe grisea. Administration for Quality and Technology Supervision of Hunan Province, Changsha, China. 7 pp.

Jiang, H., Feng, Y., Bao, L., Li, X., Gao, G., Zhang, Q., Xiao, J., $\mathrm{Xu}, \mathrm{C}$. and He, Y. 2012. Improving blast resistance of Jin 23B and its hybrid rice by marker-assisted gene pyramiding. Mol. Breed. 30:1679-1688.

Jiang, H., Feng, Y., Qiu, L., Gao, G., Zhang, Q. and He, Y. 2020. Identification of blast resistance QTLs based on two advanced backcross populations in rice. Rice 13:31.

Katsantonis, D., Kadoglidou, K., Dramalis, C. and Puigdollers, P. 2017. Rice blast forecasting models and their practical value: a review. Phytopathol. Mediterr. 56:187-216.

Liang, Y., Yan, B.-Y., Peng, Y.-L., Ji, Z.-J., Zeng, Y.-X., Wu, H.-L. and Yang, C.-D. 2017. Molecular screening of blast resistance genes in rice germplasms resistant to Magnaporthe oryzae. Rice Sci. 24:41-47.

Peng, Z., Liu, Y., He, Q., Tan, Y., Pei, Y., Deng, H. and Xing, J. 2019. Population diversity and variation of the rice blast fungus under different climate conditions. Eur. J. Plant Pathol. 155:881-889.

Raveloson, H., Ratsimiala Ramonta, I., Tharreau, D. and Sester, M. 2017. Long-term survival of blast pathogen in infected rice residues as major source of primary inoculum in high altitude upland ecology. Plant Pathol. 67:610-618.

Skamnioti, P. and Gurr, S. J. 2009. Against the grain: safeguarding rice from rice blast disease. Trends Biotechnol. 27:141150.

Sun, P., Liu, J., Wang, Y., Jiang, N., Wang, S., Dai, Y., Gao, J., Li, Z., Pan, S., Wang, D., Li, W., Liu, X., Xiao, Y., Liu, E., Wang, G.-L. and Dai, L. 2013. Molecular mapping of the blast resistance gene Pi49 in the durably resistant rice cultivar Mowanggu. Euphytica 192:45-54.

Talbot, N. J. 2003. On the trail of a cereal killer: exploring the biology of Magnaporthe grisea. Annu. Rev. Microbiol. 57:177202.

Vasudevan, K., Vera Cruz, C. M., Gruissem, W. and Bhullar, N. K. 2014. Large scale germplasm screening for identification of novel rice blast resistance sources. Front. Plant Sci. 5:505. 